\title{
Efficient truxenone-based acceptors for organic photovoltaics
}

\author{
Christian B. Nielsen, ${ }^{* a}$ Eszter Voroshazi, ${ }^{b}$ Sarah Holliday, ${ }^{a}$ Kjell Cnops, ${ }^{b, c}$ Barry P. Rand ${ }^{b}$ and Iain \\ McCulloch $^{a}$
}

\author{
Received (in XXX, XXX) Xth XXXXXXXXX 20XX, Accepted Xth XXXXXXXXX 20XX \\ ${ }_{5}$ DOI: $10.1039 / \mathbf{b 0 0 0 0 0 0 x}$
}

Two electron-deficient truxenone derivatives are designed and synthesised for organic photovoltaic applications. The promise of this class of compounds as acceptor materials is illustrated by the fabrication of efficient bilayer solar cells 10 with a subphthalocyanine (SubPc) donor, clearly outperforming reference cells with a soluble fullerene derivative as the acceptor.

Faced with the continued improvement of organic photovoltaic (OPV) devices, most materials research has been focused on the 15 development of new polymeric donors. ${ }^{1}$ These donor materials are routinely tested with phenyl-C61-butyric acid methyl ester (PCBM) or closely related fullerene derivatives and solar cell efficiencies are fast approaching $10 \% .^{2}$ A common theme of this research is the judicious adjustment of donor properties to obtain 20 an advantageous interaction, both energetically and morphologically, with the fullerene acceptor. ${ }^{3-5}$ Less effort, on the other hand, has been put into the development of new acceptor materials to complement existing or newly developed donor materials, to increase the understanding of the donor25 acceptor interactions and to address some of the disadvantages associated with fullerene acceptors such as poor optical absorption and limited solubility especially in non-halogenated solvents. ${ }^{6-10}$ In order to investigate some of these aspects, there is the possibility to explore novel electron acceptors with curved 30 isotropic electron-accepting motifs. By controlling the molecular curvature and the solubilising substituents, we aim to control the charge transport properties as well as the tendency for these small molecules to crystallise.

Here, we present two new truxenone-based acceptor materials 35 that are highly soluble in many common organic solvents and highly absorptive in most of the ultraviolet-visible region of the spectrum, complementary to the absorption spectra of the majority of low bandgap donor polymers. Our synthetic design allows for easy control of the frontier energy levels and the 40 promise of this new class of acceptors is illustrated with the fabrication of bilayer solar cells with efficiencies around $1 \%$ using subphthalocyanine (SubPc) as the donor constituent.

The synthetic route employed for the preparation of the two truxenone-based acceptors is shown in Scheme 1. Starting from ${ }_{45} 4,9,14$-tribromotruxenone, ${ }^{11}$ the two thienyl-substituted truxenones 1a-b were easily obtained in moderate yields through a Suzuki-coupling with 5-hexyl and 3-hexylthiophene-2-boronic acid pinacol ester, respectively. A subsequent Knoevenagel- condensation with malononitrile afforded the dicyanomethylene50 derivatives $\mathbf{2 a}$ and $\mathbf{2 b}$ in moderate yields. Acceptors $\mathbf{2 a - b}$ are highly soluble in both halogenated and non-halogenated solvents such as tetrahydrofuran, toluene and xylene. Thermogravimetric analysis indicated good thermal stability of both compounds (Table 1), while no clear thermal transitions were observed by 55 differential scanning calorimetry.
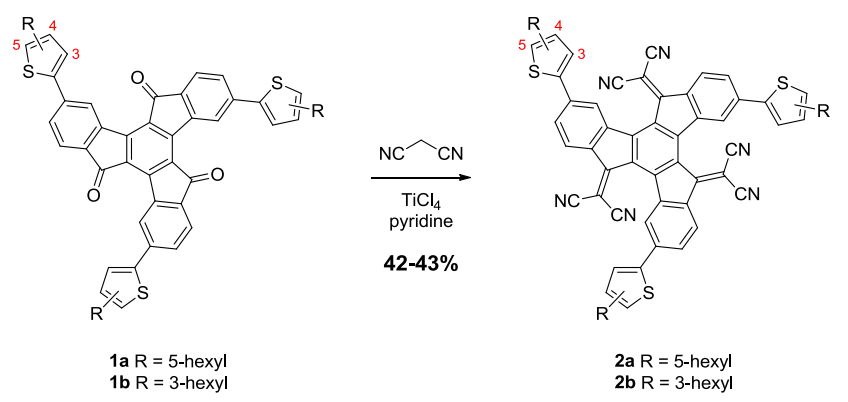

Scheme 1 Synthesis of truxenone-based acceptor compounds $\mathbf{2 a - b}$.

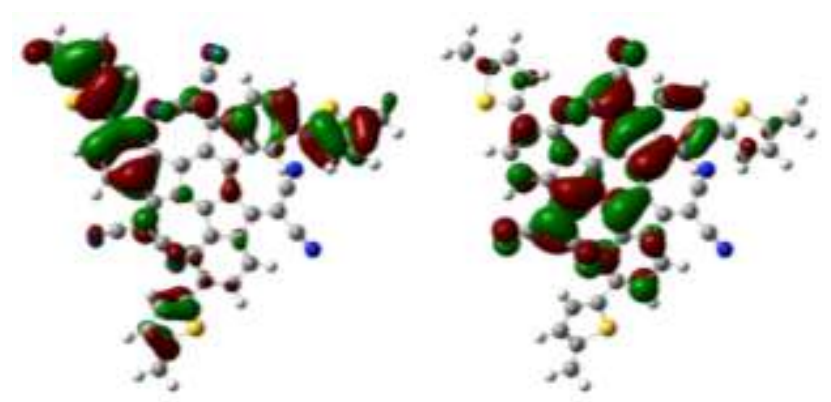

Fig. 1 Minimum-energy conformation of $\mathbf{2 b}$ (with a methyl group instead 60 of a hexyl group) calculated with Gaussian at the B3LYP/6-31G* level visualising the HOMO (left) and LUMO (right)

A computational study of the minimum-energy conformations, as illustrated for compound 2a in Figure 1, shows that the introduction of the dicyanomethylene moieties forces the truxene 65 core to adapt a bowl-shaped conformation; this finding is in agreement with crystal structures of related compounds. ${ }^{12,13}$ For 2a, the thienyl group is predicted to be twisted $26^{\circ}$ relative to the adjacent phenyl group, whereas the corresponding dihedral angle is $41^{\circ}$ for $\mathbf{2 b}$ due to the repositioning of the hexyl group. This 70 highlights the fact that the alkylated thienyl moiety can be used effectively to control the degree of conjugation as well as the tendency to crystallise through control of the torsional twist. For both acceptor molecules, the highest occupied molecular orbital 
Table 1 Thermal, optical, electrochemical and computational properties of truxenone derivatives $\mathbf{2 a - b}$.

\begin{tabular}{|c|c|c|c|c|c|c|c|c|c|}
\hline \multirow[b]{2}{*}{ Compound } & \multirow[t]{2}{*}{$\mathrm{T}_{\mathrm{d}}\left({ }^{\circ} \mathrm{C}\right)^{\mathrm{a}}$} & \multicolumn{2}{|c|}{$\lambda_{\max }(\mathrm{nm})$} & \multicolumn{2}{|c|}{ HOMO $(\mathrm{eV})$} & \multicolumn{2}{|c|}{ LUMO (eV) } & \multicolumn{2}{|c|}{$\mathrm{Eg}(\mathrm{eV})$} \\
\hline & & $\operatorname{soln}^{\mathrm{b}}$ & film ${ }^{\mathrm{c}}$ & $\exp ^{\mathrm{d}}$ & $\mathrm{calc}^{\mathrm{e}}$ & $\exp ^{\mathrm{d}}$ & $\mathrm{calc}^{\mathrm{e}}$ & $\mathrm{opt}^{\mathrm{f}}$ & $\mathrm{CV}^{\mathrm{d}}$ \\
\hline $2 \mathbf{a}$ & 338 & 298,423 & $315,451,557,602$ & -5.94 & -6.12 & -4.07 & -4.05 & 1.87 & 1.87 \\
\hline $2 \mathbf{b}$ & 340 & 287,415 & 290,423 & -6.17 & -6.29 & -4.08 & -4.16 & 2.04 & 2.09 \\
\hline РСВM & & & & -5.89 & & -3.75 & & & 2.14 \\
\hline
\end{tabular}

${ }^{a} 5 \%$ weight loss by thermogravimetric analysis. ${ }^{b}$ Dichloromethane solution. ${ }^{c}$ Spin-cast from chloroform solution $(10 \mathrm{mg} / \mathrm{ml}) .{ }^{d}$ Cyclic voltammetry in dichloromethane solution with tetrabutylammonium hexafluorophosphate electrolyte. ${ }^{e}$ Calculated with Gaussian at the B3LYP/6-31G* $\mathrm{G}^{*}$ evel. ${ }^{f}$ Determined from the onset of absorption in the solid state.

5 (HOMO, Figure 1 left) is predominantly located on the electronrich periphery, while the lowest unoccupied molecular orbital (LUMO, Figure 1 right) is distributed over the electron-poor core of the system.

Optical absorption spectroscopy supports the increased 10 torsional twist predicted for $\mathbf{2} \mathbf{b}$ relative to $\mathbf{2} \mathbf{a}$ as illustrated in Figure 2. While compound 2a shows an absorption maximum at $451 \mathrm{~nm}$ in the solid state with additional low-energy peaks at 557 and $602 \mathrm{~nm}$, compound $\mathbf{2 b}$ is significantly blue-shifted with a $\lambda_{\max }$ at $423 \mathrm{~nm}$. We moreover find that $\mathbf{2 a}$ displays a significantly 15 larger red-shift than $\mathbf{2 b}$ upon transition from solution to the solid state as evident from the data in Table 1. The optical band gap is $1.87 \mathrm{eV}$ for $\mathbf{2 a}$ and $2.04 \mathrm{eV}$ for $\mathbf{2} \mathbf{b}$, which again is in agreement with the reduced conjugation caused by the twisted thiophene.

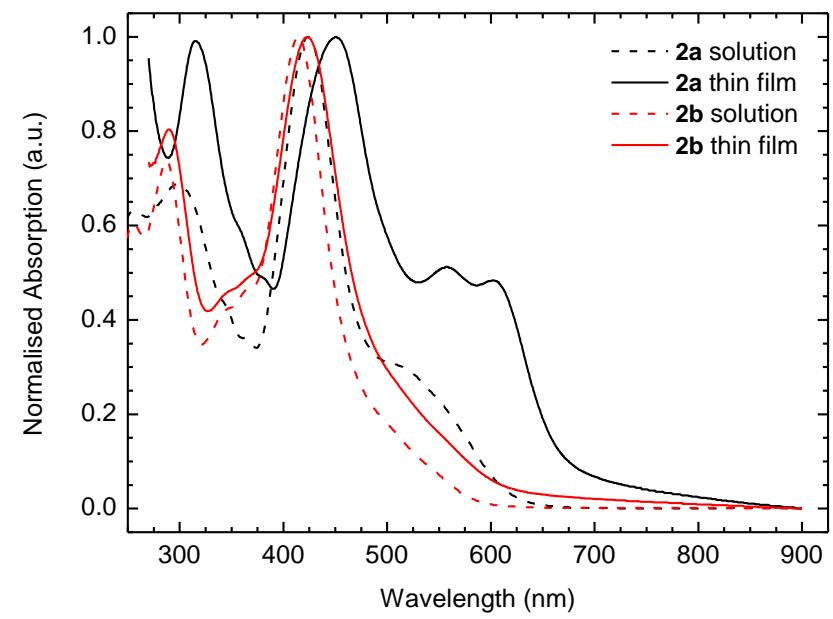

Fig. 2 Normalised UV-vis absorption spectra of $\mathbf{2 a}$ and $\mathbf{2 b}$ in dichloromethane solution and as spin-cast films from chloroform.

In order to assess the frontier energy levels of compounds 2ab, electrochemical characterisation was carried out by means of cyclic voltammetry (CV). The HOMO and LUMO energy levels 25 were estimated from the onsets of oxidation and reduction, respectively (Table 1). The HOMO levels were found to be -5.94 $\mathrm{eV}$ for $\mathbf{2 a}$ and $-6.17 \mathrm{eV}$ for $\mathbf{2 b}$, which is in good agreement with the predicted values from the computational study. The electronrich 5-hexyl-2-thienyl substituent in compound 2a is less twisted 30 than the 3-hexyl-2-thienyl substituent (2b) and will therefore provide a higher degree of $\pi$-conjugation and thus a higher-lying HOMO level. The LUMO levels, on the other hand, are found to be almost identical for the two compounds; $-4.07 \mathrm{eV}$ for $\mathbf{2 a}$ and $4.08 \mathrm{eV}$ for $\mathbf{2 b}$. These results corroborate nicely the assumption 35 from the computational study that the HOMO level is mainly governed by the peripheral substituents, while the LUMO level is largely controlled by the electron-poor dicyanomethylenefunctionalised truxenone core, which is identical for the two compounds. For comparison under identical conditions, we also 40 tested PCBM by CV and found a HOMO level of $-5.89 \mathrm{eV}$ and a LUMO level of $-3.75 \mathrm{eV}$ indicating that the two truxenone-based acceptors presented herein have slightly larger electron affinities than PCBM.

Acceptor compounds $\mathbf{2 a}$ and $\mathbf{2 b}$ were tested in inverted bilayer 45 solar cells fabricated with a vacuum-deposited donor material in conjunction with our solution-processed truxenone-based acceptor materials. Devices with inverted architectures were prepared as this allowed for solution-deposition of the truxenone acceptors prior to vacuum-deposition of the donor materials. The 50 bilayer device configuration was chosen over a bulkheterojunction device architecture in order to eliminate any potential morphology-related issues that could obscure evaluation of the OPV properties of this new class of acceptor compounds. As donor material in the bilayer devices, we investigated both a 55 wide band gap donor, SubPc with HOMO/LUMO levels of -5.5/$3.4 \mathrm{eV}$ and a narrower band gap material, zinc phthalocyanine (ZnPc) with HOMO/LUMO levels of $-5.2 /-3.5 \mathrm{eV} .^{14,15}$

Prior to device preparation, the topography of the truxenone thin films was characterised by atomic force microscopy (Fig. ${ }_{60} \mathrm{~S} 10$ ). Compound 2a forms ordered features (root mean square $(\mathrm{rms})$ roughness $=5.3 \mathrm{~nm})$ in agreement with the large red-shift in absorption upon transition from solution to the solid state indicative of aggregation and/or crystallisation. In contrast, films of $\mathbf{2 b}$ are featureless and smooth ( $\mathrm{rms}$ roughness $=0.6 \mathrm{~nm}$ ), 65 suggesting an amorphous film.

Photovoltaic parameters of the best devices are listed in Table 2 and corresponding current-voltage characteristics are shown in Fig. S11 and S12. Bilayer devices comprising SubPc and 2a or 2b achieve short-circuit current densities $\left(J_{S C}\right)$ around $2 \mathrm{~mA} / \mathrm{cm}^{2}$, 70 which is comparable to the $J_{S C}$ obtained for reference cells with PCBM. Bilayer devices with $\mathrm{ZnPc}$ and $\mathbf{2 a}$ or $\mathbf{2 b}$ have a $J_{S C}$ of 2.4 $\mathrm{mA} / \mathrm{cm}^{2}$ and $1.9 \mathrm{~mA} / \mathrm{cm} 2$, respectively, whereas the PCBMbased device displays a higher $J_{S C}$ of $3.0 \mathrm{~mA} / \mathrm{cm}^{2}$. Complementary and red-shifted absorption (Fig. 3) of $\mathrm{ZnPc}$ 75 explain the higher $J_{S C^{-}}$values obtained for the $\mathrm{ZnPc}$ cells in comparison to the SubPc cells. External quantum efficiency (EQE) spectra of the devices as shown in Fig. 3 indicate that both donor ( $\mathrm{SubPc}$ and $\mathrm{ZnPc}$ ) and acceptor (2a and $\mathbf{2 b}$ ) compounds contribute to the photocurrent. This confirms that the energy level 80 alignment at the heterojunction permits an efficient separation of the bound geminate pairs generated both on the truxenone acceptor and $\mathrm{SubPc}$ or $\mathrm{ZnPc}$ layers. $J_{S C}$-values extracted from the EQE spectra by integration (Table 2) correlate well with the values obtained from the $J-V$ measurements. The open-circuit 85 voltage $\left(V_{O C}\right)$ of the SubPc devices with $2 \mathbf{a}(947 \mathrm{mV})$ and $2 \mathbf{b}$ $(923 \mathrm{mV})$ is approximately $100 \mathrm{mV}$ lower than the value obtained 
with PCBM; this observation is in agreement with the lower lying LUMO levels of the truxenones reported in Table 1. Owing to a fill factor (FF) of $52 \%$, the $\mathbf{2 a}$ /SubPc device reaches a power conversion efficiency (PCE) of $1.0 \%$, outperforming the PCBM 5 reference device, which has a FF of $34 \%$ and a PCE of $0.8 \%$. The $\mathbf{2 b} /$ SubPc device performs similarly to the PCBM reference device with a FF of $36 \%$ and a PCE of $0.7 \%$.

Table 2 Photovoltaic properties of bilayer solar cells with SubPc ${ }^{\mathrm{a}}$

\begin{tabular}{ccccccc}
\hline Acceptor & Donor & $\begin{array}{c}\mathrm{V}_{\mathrm{oc}} \\
(\mathrm{mV})\end{array}$ & $\begin{array}{c}\mathrm{J}_{\mathrm{sc}} \\
\left(\mathrm{mA} / \mathrm{cm}^{2}\right)\end{array}$ & $\begin{array}{c}\mathrm{J}_{\mathrm{sc}}(\mathrm{EQE}) \\
\left(\mathrm{mA} / \mathrm{cm}^{2}\right)\end{array}$ & $\begin{array}{c}\mathrm{FF} \\
(\%)\end{array}$ & $\begin{array}{c}\text { PCE } \\
(\%)\end{array}$ \\
2a & SubPc & 947 & 1.9 & 1.8 & 52 & 1.0 \\
2b & & 923 & 2.0 & 1.7 & 36 & 0.7 \\
PCBM & & 1060 & 2.1 & 1.6 & 34 & 0.8 \\
2a & $\mathrm{ZnPc}$ & 448 & 2.4 & 1.8 & 50 & 0.6 \\
$\mathbf{2 b}$ & & 276 & 1.4 & 1.2 & 28 & 0.1 \\
PCBM & & 612 & 3.0 & 2.9 & 61 & 1.1
\end{tabular}

${ }^{a}$ Device architecture and thicknesses: ITO/ZnO $(10 \mathrm{~nm}) / \mathbf{2 a}(65-70 \mathrm{~nm})$ 10 or $\mathbf{2 b}(50 \mathrm{~nm})$ or PCBM $(10 \mathrm{~nm}) / \mathrm{SubPc}(30 \mathrm{~nm})$ or $\mathrm{ZnPc}(50 \mathrm{~nm}) / \mathrm{MoO}_{3}$ $(5 \mathrm{~nm}) / \mathrm{Ag}(120 \mathrm{~nm})$.

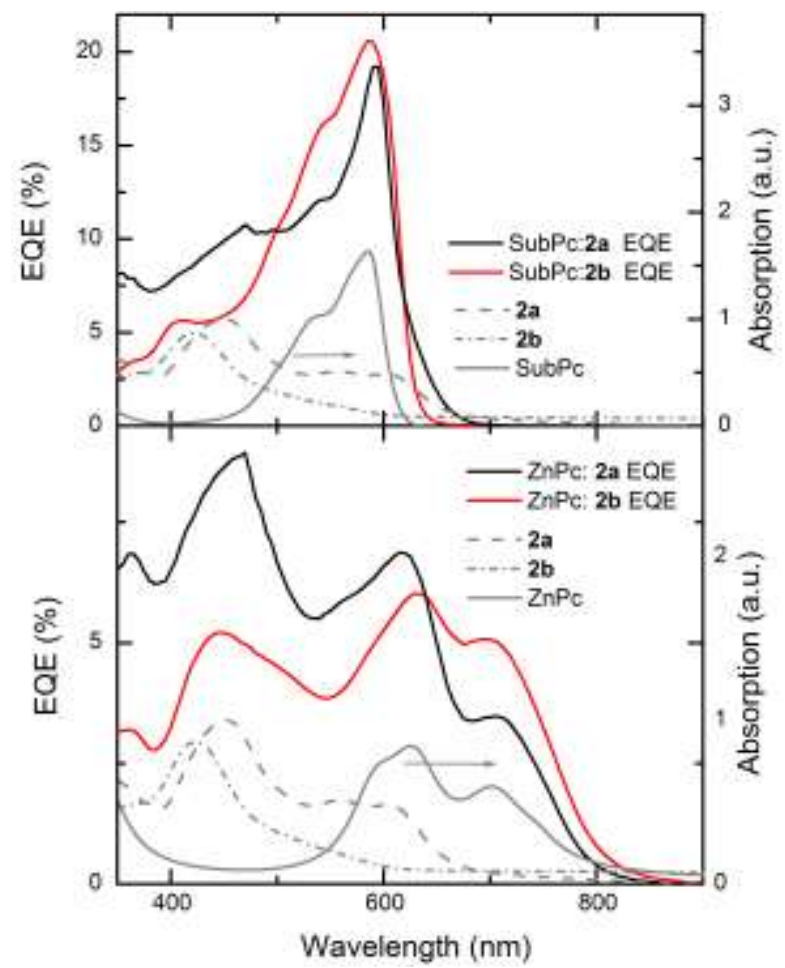

Fig. 3. External quantum efficiency (EQE) of $\mathbf{2 a}$ or $\mathbf{2} \mathbf{b} / \mathrm{SubPc}$ (top panel) 15 and $\mathbf{2 a}$ or $\mathbf{2} \mathbf{b} / \mathrm{ZnPc}$ (bottom panel) devices and absorption spectra of the various compounds.

In contrast to the truxenone/SubPc devices, the truxenone/ZnPc bilayer devices display significantly reduced $V_{O C}$-values in comparison to the PCBM reference device. 20 Although the truxenone-based devices have elevated leakage currents (Fig. S12), it cannot explain entirely the reduced $V_{O C}$. Altered growth of the donor molecules on the different acceptor layers might also influence the energy levels of $\mathrm{ZnPc}$ and, consequently, reduce the $V_{O C}{ }^{15}$ Truxenone derivative $\mathbf{2 a}$ affords 25 a $V_{O C}$ of $448 \mathrm{mV}$, which in conjunction with a FF of $50 \%$, results in a moderate PCE of $0.6 \%$, while a much lower $V_{O C}$ of $276 \mathrm{mV}$ and a lower FF of $28 \%$ is responsible for a low PCE-value of
$0.1 \%$ for $\mathbf{2 b}$. Both truxenone-based acceptors in this case show inferior performance to the PCBM reference device, which has a ${ }_{30} \mathrm{PCE}$ of $1.1 \%$ due to a higher $V_{O C}$ of $612 \mathrm{mV}$ and a FF of $60 \%$. It is notable that, with both donor materials, devices with 2a have higher FFs than devices with $\mathbf{2} \mathbf{b}$. This suggests that $\mathbf{2 a}$, with the nearly coplanar 5-hexyl-2-thienyl substituents, is likely to have a higher charge carrier mobility than $\mathbf{2 b}$, which has the more 35 twisted 3-hexyl-2-thienyl substitution pattern.

In conclusion, we have developed two new truxenone-based acceptor materials (2a and $\mathbf{2 b}$ ) and shown how chemical modification of the central truxenone core can be used to adjust the LUMO level, while chemical modification of the peripheral 40 substituents can be used to adjust the HOMO level without further affecting the LUMO level. Additionally, the peripheral substituent can very effectively be used to control the degree of torsional twist and hence the degree of intermolecular aggregation.

45 The two new acceptors were proven to work satisfactorily in bilayer photovoltaic devices with two common donor materials, SubPc and ZnPc. In both cases, acceptor 2a performs significantly better than $\mathbf{2 b}$ and with a SubPc donor material compound 2a even outperforms the PCBM acceptor, giving a ${ }_{50} 25 \%$ higher power conversion efficiency. This initial screening clearly suggests that this novel class of acceptors has potential in photovoltaic applications. However, both thin film deposition and growth of the donor materials could be further optimized to reach higher performance. Further device characterisation could also 55 yield insight into the efficiency of the photogeneration process and eventual loss mechanisms limiting the performance of $\mathrm{ZnPc/truxenone} \mathrm{devices.}$

This work was in part carried out with financial support from SUPERGEN, EC FP7 Project X10D and EC FP7 Project ONE-P 60 with support from BASF.

\section{Notes and references}

${ }^{a}$ Department of Chemistry and Centre for Plastic Electronics, Imperial College London, London SW7 2AZ, U.K. E-mail: c.nielsen@imperial.ac.uk

$65{ }^{b}$ IMEC, Kapeldreef 75, B-3001 Heverlee, Belgium.

${ }^{c}$ Katholieke Universiteit Leuven, ESAT, Kasteelpark Arenberg 10,

Heverlee, Belgium.

$\dagger$ Electronic Supplementary Information (ESI) available: Experimental details, NMR spectra, TGA and DSC traces, cyclic voltammograms,

70 AFM micrographs, J-V curves and photoluminescence spectra. See DOI: $10.1039 / \mathrm{b} 000000 \mathrm{x} /$

1. C. L. Chochos and S. A. Choulis, Prog. Polym. Sci., 2011, 36, 13261414.

2. Z. He, C. Zhong, S. Su, M. Xu, H. Wu and Y. Cao, Nat Photon, $75 \quad$ 2012, 6, 593-597.

3. M. C. Scharber, D. Mühlbacher, M. Koppe, P. Denk, C. Waldauf, A. J. Heeger and C. J. Brabec, Adv. Mater., 2006, 18, 789-794.

4. J. Kirkpatrick, C. B. Nielsen, W. Zhang, H. Bronstein, R. S. Ashraf, M. Heeney and I. McCulloch, Adv. Energy Mater., 2012, 2, 260-265.

80 5. C. Nielsen, R. Ashraf, B. Schroeder, P. D'Angelo, S. E. Watkins, K Song, T. D. Anthopoulos and I. McCulloch, Chem. Commun., 2012.

6. J. E. Anthony, Chem. Mater., 2011, 23, 583-590.

7. S. Shoaee, T. M. Clarke, C. Huang, S. Barlow, S. R. Marder, M. Heeney, I. McCulloch and J. R. Durrant, J. Am. Chem. Soc., 2010, 132, 12919-12926.

8. J. T. Bloking, X. Han, A. T. Higgs, J. P. Kastrop, L. Pandey, J. E. Norton, C. Risko, C. E. Chen, J.-L. Brédas, M. D. McGehee and A. Sellinger, Chem. Mater., 2011, 23, 5484-5490. 
9. G. Ren, E. Ahmed and S. A. Jenekhe, Adv. Energy Mater., 2011, 1, 946-953.

10. Y. Zhou, L. Ding, K. Shi, Y.-Z. Dai, N. Ai, J. Wang and J. Pei, Adv. Mater., 2012, 24, 957-961.

5 11. C. Lambert, G. Nöll, E. Schmälzlin, K. Meerholz and C. Bräuchle, Chem.--Eur. J., 1998, 4, 2129-2135.

12. K. Jacob, J. Y. Becker, A. Ellern and V. Khodorkovsky, Tetrahedron Lett., 1999, 40, 8625-8628.

13. E. M. Pérez, M. Sierra, L. Sánchez, M. R. Torres, R. Viruela, P. M.

10 Viruela, E. Ortí and N. Martín, Angew. Chem. Int. Ed., 2007, 46, 1847-1851.

14. P. Heremans, D. Cheyns and B. P. Rand, Acc. Chem. Res., 2009, 42, 1740-1747.

15. B. P. Rand, D. Cheyns, K. Vasseur, N. C. Giebink, S. Mothy, Y. Yi,

15 V. Coropceanu, D. Beljonne, J. Cornil, J.-L. Brédas and J. Genoe, Adv. Funct. Mater., 2012, 22, 2987-2995. 